\title{
The inner gaseous accretion disk around a Herbig Be star revealed by near- and mid-infrared spectro-interferometry
}

\author{
S. Kraus, Th. Preibisch and K. Ohnaka \\ Max-Planck-Institut für Radioastronomie, Auf dem Hügel 69, 53121 Bonn, Germany \\ email: skraus@mpifr-bonn.mpg.de
}

\begin{abstract}
Herbig Ae/Be stars are pre-main-sequence stars of intermediate mass, which are still accreting material from their environment, probably via a disk composed of gas and dust. Here we present a recent study of the geometry of the inner (AU-scale) circumstellar region around the Herbig Be star MWC 147 using long-baseline interferometry. By combining for the first time near- and mid-infrared spectro-interferometry on a Herbig star, our VLTI/AMBER and VLTI/MIDI data constrain not only the geometry of the brightness distribution, but also the radial temperature distribution in the disk. The emission from MWC 147 is clearly resolved and has a characteristic physical size of $\sim 1.3 \mathrm{AU}$ and $\sim 9 \mathrm{AU}$ at $2.2 \mu \mathrm{m}$ and $11 \mu \mathrm{m}$ respectively. This increase in apparent size towards longer wavelengths is much steeper than predicted by analytic disk models assuming power-law radial temperature distributions. For a detailed modeling of the interferometric data and the spectral energy distribution of MWC 147, we employ 2-D frequency-dependent radiation transfer simulations. This analysis shows that passive irradiated Keplerian dust disks can easily fit the SED, but predict much lower visibilities than observed, so these models can clearly be ruled out. Models of a Keplerian disk with emission from an optically thick inner gaseous accretion disk (inside the dust sublimation zone), however, yield a good fit of the SED and simultaneously reproduce the observed near- and mid-infrared visibilities. We conclude that the near-infrared continuum emission from MWC 147 is dominated by accretion luminosity emerging from an optically thick inner gaseous disk, while the mid-infrared emission also contains strong contributions from the passive irradiated dust disk.
\end{abstract}

Keywords. Stars: formation, pre-main-sequence, individual (MWC 147), accretion, accretion disks, techniques: interferometric, radiative transfer.

\section{Introduction}

The spatial structure of the circumstellar material around Herbig Ae/Be stars, i.e. intermediate-mass pre-main sequence stars, is still a matter of debate. Until recently, the spatial scales of the inner circumstellar environment (a few AU, corresponding to $\lesssim 0.1$ arcsecond) were not accessible to optical and infrared imaging observations, and conclusions drawn on the spatial distribution of the circumstellar material were, in most cases, entirely based on the modeling of the spectral energy distribution (SED). However, as demonstrated by Men'shchikov \& Henning (1997) and others, these SED model fits are highly ambiguous, and only the combination of SED modeling with high-resolution imaging can provide crucial constraints on the real geometry of the circumstellar matter. Since these ambiguities concern not only the disk geometry, but also the general nature of the disk (e.g. actively accreting vs. passive irradiated disks), high angular resolution observations are urgently required.

In recent years, near-infrared (NIR) interferometry could make important contributions for a better understanding of the structure of the circumstellar material around 


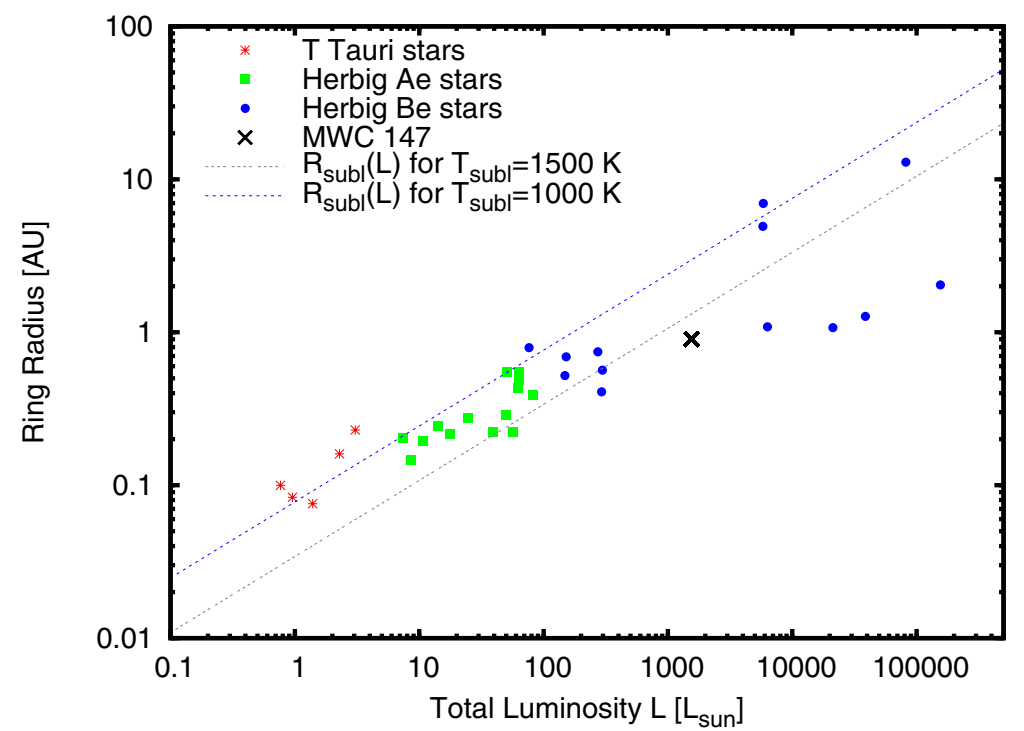

Figure 1. Size-luminosity relation for NIR long-baseline interferometric measurements on YSOs (adopted from Millan-Gabet et al. 2007). While the measured sizes for most T Tauri and Herbig Ae stars agree to the dust sublimation radius $R_{\text {subl }}$ expected for sublimation temperatures between 1000 and $1500 \mathrm{~K}$ (dotted lines), some Herbig Be stars (including MWC 147, marked as cross) show significantly smaller diameters.

these stars (see also the contribution by F. Malbet for a review). A survey performed by Millan-Gabet, Schloerb \& Traub (2001) with the IOTA interferometer showed that the near-infrared continuum emission is best described by ring-like or spherical geometries rather than with the "classical" optically thick, geometrically thin accretion disk models (e.g. Lynden-Bell \& Pringle 1974; Hillenbrand et al. 1992). Later, a correlation between the NIR size and the stellar luminosity was found (Fig. 1; see Monnier \& MillanGabet 2002), which suggests that the NIR continuum emission mainly traces hot dust at the inner sublimation radius, missing any shielding by optically thick gas inside of the sublimation radius. This observational result also stimulated theoretical work, especially for passive irradiated circumstellar disks, e.g. by Natta, Prusti, Neri, et al. (2001) and Dullemond, Dominik \& Natta (2001). These models reproduce the ring-like morphology by introducing pronounced, hot dust emission at the inner rim of the circumstellar disk.

However, for the more luminous Herbig Be stars, deviations from the simple $R \propto L^{1 / 2}$ size-luminosity relation were found (see Fig. 1), indicating that for these hot stars, the inner rim lies closer to the star than expected from the stellar luminosity. Monnier \& Millan-Gabet (2002) suggested that this might be due to the presence of an inner gaseous disk, which shields the dust disk from the strong stellar ultraviolet (UV) radiation. Since this shielding would be most efficient for hot stars, it would allow the inner rim of the dust disk around B-type stars to exist closer to the star. Several subsequent studies favor classical accretion disk models (e.g. Eisner et al. 2004; Monnier et al. 2005), in which the infrared emission contains contributions from the thermal emission of optically thick gas in the innermost disk regions.

Whereas the majority of the existing infrared interferometric studies on YSOs were performed at NIR wavelengths ( $H$ and $K$-band), a smaller number of studies investigated also their mid-infrared (MIR) ( $N$-band) emission (e.g. Leinert et al. 2004; Preibisch et al. 2006). The latest generation of interferometric instruments provides not only the high 
spatial resolution achievable with long-baseline interferometry, but also some spectroscopic capabilities. In these cases, the interferograms are spectrally dispersed, such that the fringe contrast (visibility) can be measured for individual spectral channels.

Considering a simple model where each disk annulus radiates as a black-body of different temperature, it is clear that different spectral channels probe different physical regions within the disk. Since spectro-interferometry allows us to measure the spatial geometry (in first approximation the object extension) in each spectral channel, these measurements constrain simultaneously the geometry and also the radial temperature distribution in the disk. In general, one expects the NIR emission to originate mainly from the hottest dust located close to the dust sublimation radius, with significant contributions from the stellar photosphere and/or from scattered light. MIR wavelengths trace also slightly colder dust located further out in the disk. Whereas simple analytic models for the disk temperature distribution provide some understanding for the general dependences, more sophisticated radiative transfer simulations are required to include dust scattering and optical depth effects.

\section{Interferometric observations of MWC 147}

MWC 147 is a well studied intermediate-mass pre-main sequence star located at a distance of 800 pc in Monoceros. For our modeling, we adopted the stellar parameters by Hernández, Calvet, Briceño et al. (2004), namely a spectral type of B6, $L_{\star}=1550 L_{\odot}$, $M_{\star}=6.6 M_{\odot}$, and $R_{\star}=6.63 R_{\odot}$. The object shows a strong infrared excess of about 6 mag at mid-infrared wavelengths, demonstrating the presence of circumstellar material. Numerous recent observational results strongly suggest the presence of a massive circumstellar disk around MWC 147. Hillenbrand, Strom, Vrba, et al. (1992) fitted the spectral energy distribution of MWC 147 with a model assuming a massive accretion disk and estimated an accretion rate of $10^{-5} M_{\odot} \mathrm{yr}^{-1}$.

First infrared interferometric observations by Akeson, Ciardi, van Belle, et al. (2000) revealed that the $K$-band size is surprisingly compact $(2.28$ mas $=0.7 \mathrm{AU}$, uniform disk diameter). We observed MWC 147 with the interferometric instruments MIDI and AMBER at the ESO Very Large Telescope Interferometer (VLTI); all details are described in Kraus, Preibisch, \& Ohnaka (2007). From the resulting seven MIDI and one AMBER measurements, we derive wavelength-dependent visibilities, which can either be fitted directly to model images or used to compute the characteristic size of the emitting region (assuming a certain intensity profile, see Fig. 2). For our fits, we included also additional $K$-broadband data from the PTI archive, and the upper limit on the $H$-band size derived by Millan-Gabet, Schloerb \& Traub (2001).

As a first step of analysis we compared the interferometric data to analytic disk models. Models of both, passive irradiated circumstellar disks as well as of active viscously accreting disks (Lynden-Bell \& Pringle 1974), predict that the radial temperature profile of YSO disks should follow a simple power-law $T(r) \propto r^{-\alpha}$. Most studies infer a power law index of $\alpha=3 / 4$ (e.g. Millan-Gabet et al. 2001) or 1/2 (e.g. Leinert et al. 2004).

Using the assumption that each disk annulus radiates as a blackbody and a temperature of $T_{\text {subl }}=1500 \mathrm{~K}$ at the inner disk truncation radius, we can compute the wavelength-dependence of the disk size corresponding to these analytic model. Adjusting the resulting wavelength-dependent size to fit the NIR size measured on MWC 147 (see Fig. 2), we find that these analytic models can not reproduce the measured NIR and MIR-sizes simultaneously. Therefore, we performed a more detailed radiative transfer modeling. 


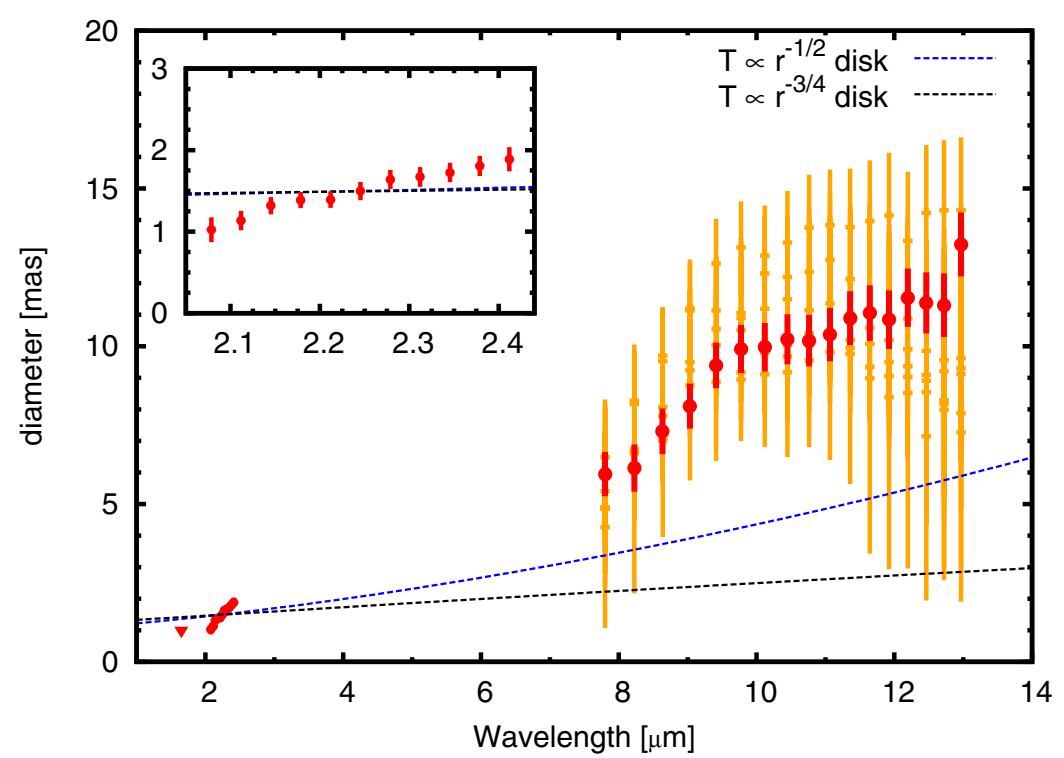

Figure 2. Wavelength-dependence of the measured characteristic size over the $H$-, $K$ - and $N$-band. Gaussian intensity profiles were assumed. For comparison, we show the wavelength-dependent size as predicted by simple analytic disk models. As the scaling of these models is arbitrary, we normalize them to the measured NIR size. It is evident that these analytic models can not describe the measured wavelength-dependent size well.

\section{2-D radiative transfer modeling}

For our modeling of the interferometric data of MWC 147 we employ the radiative transfer code mcsim_mpi (Ohnaka et al. 2006), which solves the radiative transfer problem self-consistently using a Monte Carlo approach. For each radiative transfer model, we first check the agreement with the SED of MWC 147, which we constrain using an archival Spitzer-IRS spectrum as well as photometric data from the literature. Then, a ray-trace program is used to compute synthetic images for any wavelength of interest. Finally, visibilities are computed from the simulated images for the points of the $u v$-plane covered by the data. Both the disk inclination as well as the orientation of the disk on the sky are treated as free parameters, which we fit in order to find best agreement with the spectro-interferometric visibilities. All details of the modeling are described in Kraus, Preibisch, \& Ohnaka (2007).

The dust density distribution of the accretion disk in our models resembles a flared, Keplerian rotating disk with puffed-up inner rim, as parameterized by Dullemond, Dominik \& Natta (2001) and extends from the dust sublimation radius (at $\sim 2.7 \mathrm{AU}$, assuming $T_{\text {subl }}=1500 \mathrm{~K}$ ) to $100 \mathrm{AU}$. The radial density distribution was chosen according to $\rho(r) \propto r^{-3 / 2}$ and the disk flaring index $\beta=1.175$. In order to reproduce the shape of the SED, we found that, in addition to the disk, an extended envelope is required, for which we use a density distribution of the form $\rho(r) \propto r^{-1 / 2}$. The presence of such an envelope is also supported by mid-infrared imaging by Polomski, Telesco, Piña, et al. (2002), which revealed an extended, elongated structure.

Fig. 3 shows model images, the SED, as well as the visibilities corresponding to the model of a passive, irradiated accretion disk. We find that the predicted model visibilities (especially in the NIR, but also, to a lower degree, in the MIR) are always much smaller than the measured visibilities. We conclude that although passive irradiated circumstellar 
a) Image $K$-band

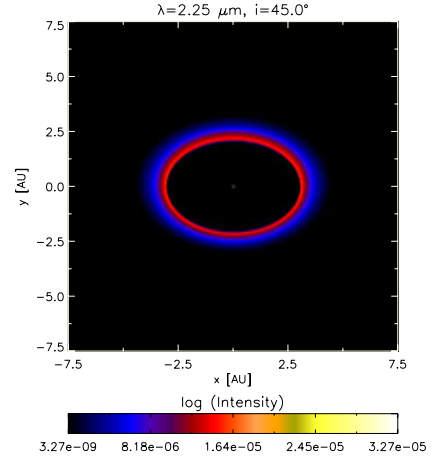

c) SED (different incl.)

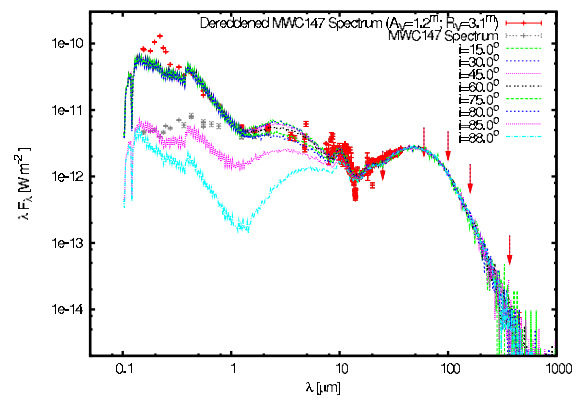

e) Visibilities NIR (IOTA/PTI)

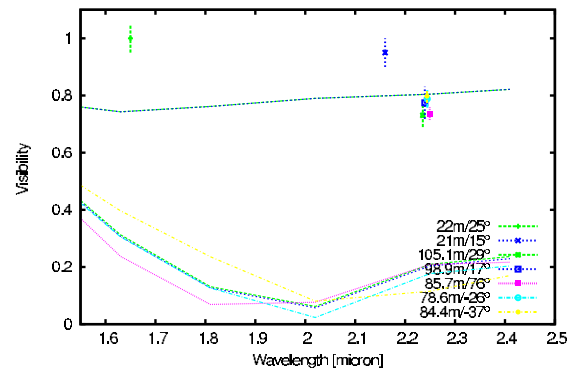

g) Visibilities MIR (MIDI)

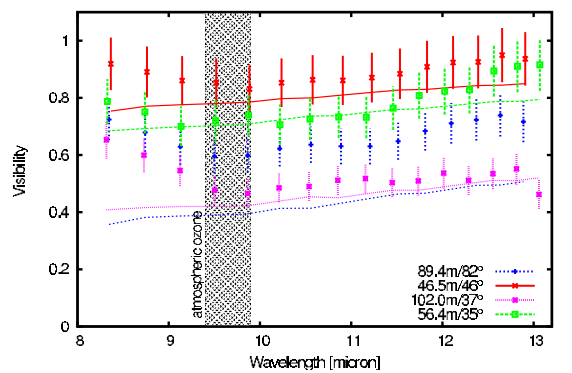

b) Image $N$-band

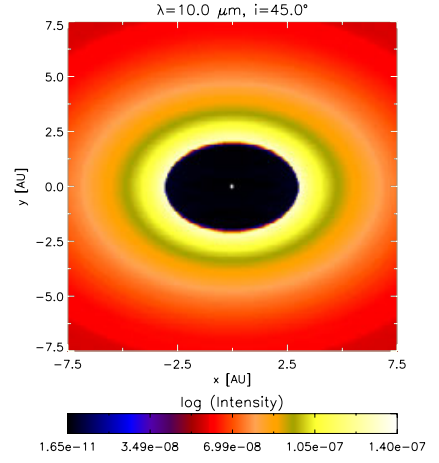

d) SED (best-fit incl. $i=45^{\circ}$ )

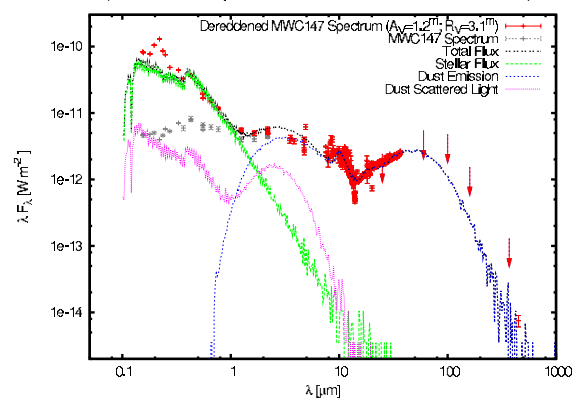

f) Visibilities NIR (AMBER)

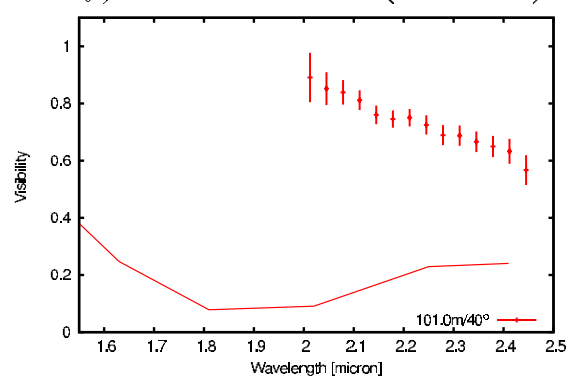

h) Visibilities MIR (MWI)

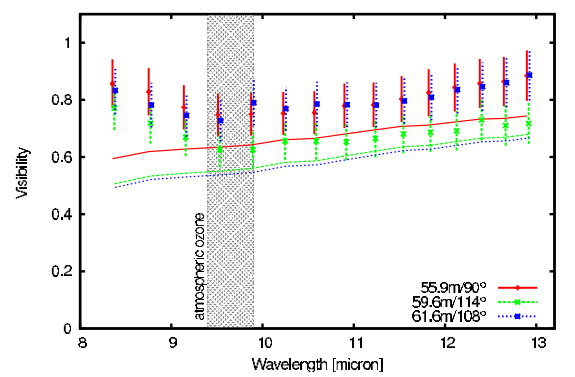

Figure 3. Best-fit radiative transfer model assuming a flared Keplerian disk geometry. This model results in a poor $\chi_{r}^{2}$ of 42 . Panels $a$ ) and $b$ ) show the ray-traced images for two representative wavelengths $(2.25 \mu \mathrm{m}$ and $10.0 \mu \mathrm{m}), c)$ shows the SED for various inclination angles, whereas $d$ ) gives the SED for the best-fit inclination angle $\left(45^{\circ}\right)$. Panels $e$ ) to $h$ ) show the observed and the model NIR and MIR visibilities. 
a) Image $K$-band

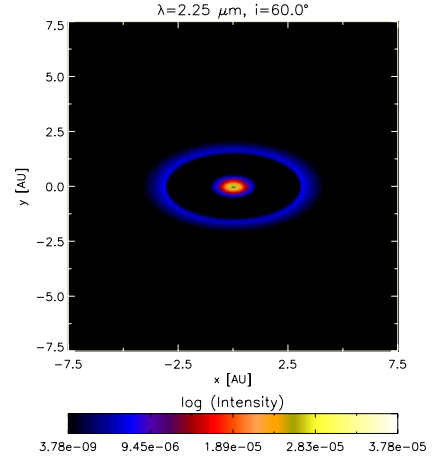

c) SED (different incl.)

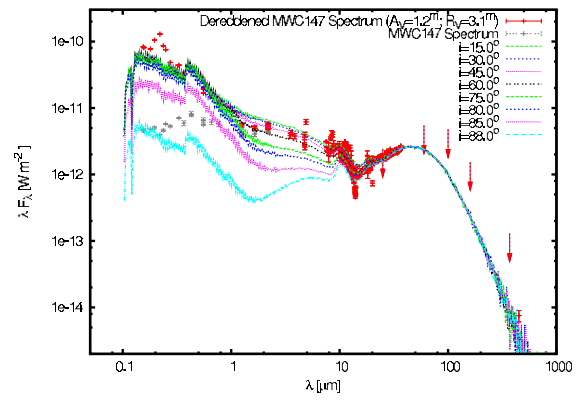

e) Visibilities NIR (IOTA/PTI)

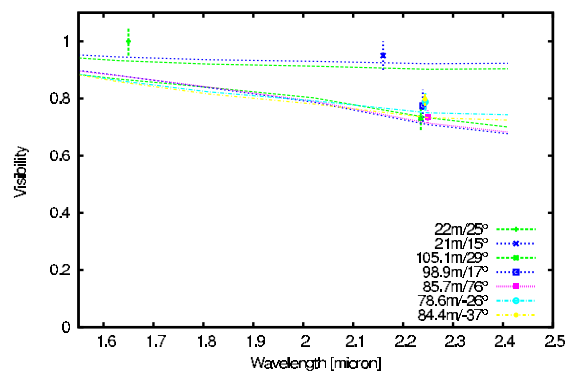

g) Visibilities MIR (MIDI)

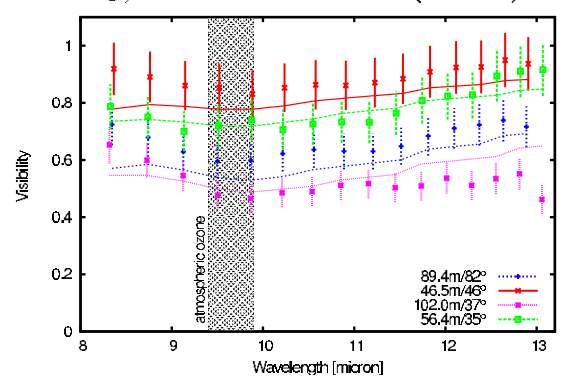

b) Image $N$-band

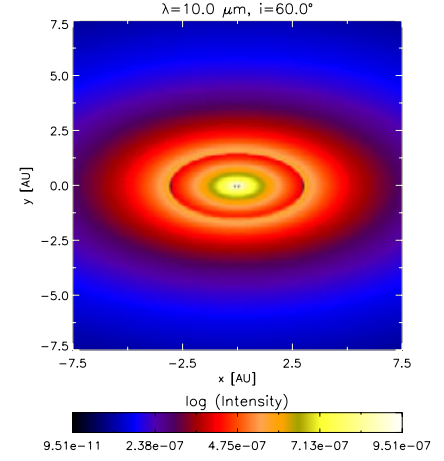

d) SED (best-fit incl. $i=60^{\circ}$ )

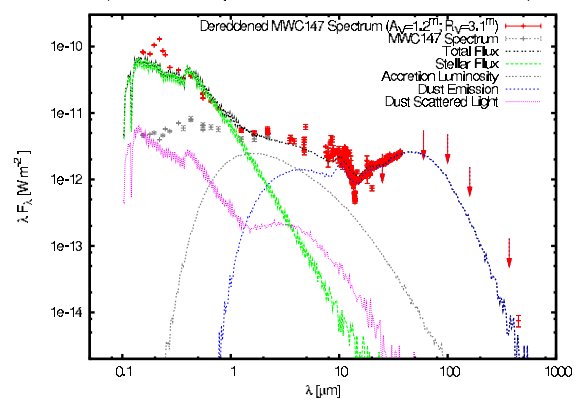

f) Visibilities NIR (AMBER)

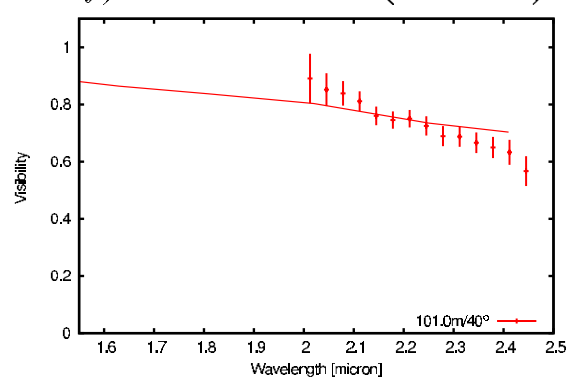

h) Visibilities MIR (MWI)

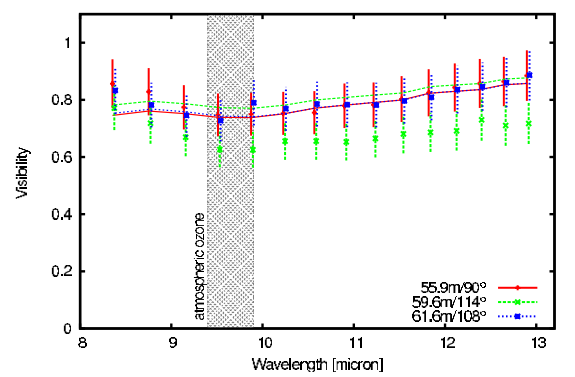

Figure 4. As Fig. 3, but for our best-fit model of a flared Keplerian disk with optically thick gas located inside the dust sublimation radius. With $\chi_{r}^{2}=1.26$ (inclination $60^{\circ}$ ), this model fits our spectro-interferometric data much better than the model without optically thick gas emission (Fig. 3). 
disk models are able to reproduce the SED of MWC 147, these models are in strong conflict with the interferometric measurements, resulting in $\chi_{r}^{2} \approx 43$.

In passive circumstellar disks, the infrared emission is generally assumed to originate almost entirely from dust; the emissivity of the inner, dust-free gaseous part of the disk, at radii smaller than the dust sublimation radius, is negligible. In an actively accreting disk, on the other hand, viscous dissipation of energy in the inner dust-free gaseous part of the accretion disk can heat the gas to high temperatures and give rise to significant amounts of infrared emission from optically thick gas. The inner edge of this gas accretion disk is expected to be located a few stellar radii above the stellar surface, where the hot gas is thought to be channeled towards the star via magnetospheric accretion columns. While the magnetospheric accretion columns are too small to be resolved in our interferometric data ( $3 R_{\star}$ correspond to $0.09 \mathrm{AU}$ or 0.12 mas), infrared emission from hot gas between the dust sublimation radius and the stellar surface should be clearly distinguishable from the thermal emission of the dusty disk due to the different temperatures of these components and the resulting characteristic slope in the NIR- and MIR-visibilities.

As MWC 147 is a quite strong accretor $\left(\dot{M}_{\mathrm{acc}} \approx 10^{-5} M_{\odot} \mathrm{yr}^{-1}\right.$; Hillenbrand et al. 1992), significant infrared emission from the inner gaseous accretion disk is expected. Muzerolle, D'Alessio, Calvet, et al. (2004) found that, even for smaller accretion rates, the gaseous inner accretion disk is several times thinner than the puffed-up inner dust disk wall and is optically thick (both in radial as well as in the vertical direction).

In order to add the thermal emission from the inner gaseous disk to our radiative transfer models, we assume the radial temperature power-law by Pringle (1981). Including the accretion luminosity from an inner gaseous disk to the model improves the agreement between model predictions and observed visibilities strongly. With a flared disk geometry and an accretion rate of $\dot{M}_{\text {acc }}=9 \times 10^{-6} M_{\odot} \mathrm{yr}^{-1}$, both the SED and the interferometric visibilities are reasonably well reproduced $\left(\chi_{r}^{2}=1.26\right.$, see Fig. 4$)$.

\section{Conclusions}

Our infrared long-baseline interferometric observations of MWC 147, constrain, for the first time, the inner circumstellar environment around a Herbig Be star over the wavelength range from 2 to $13 \mu \mathrm{m}$. We measure a strong increase of the apparent size with wavelength, with a slope much steeper than predicted by the commonly used analytic temperature power-law models. To test whether more realistic physical models of the circumstellar dust environment yield better agreement, we employed 2-D radiative transfer modeling. These models include a dust disk embedded in an extended dust envelope, and were used to simultaneously fit the SED and the spectro-interferometric observables. While models of passive irradiated disks are able to reproduce the SED, they are in strong conflict with the interferometric observables, significantly overestimating the size of both the NIR and MIR emission. Adding an inner gaseous accretion disk component to the model solves the discrepancies and provides good agreement with the interferometric data. The best-fit was obtained with a flared Keplerian disk which is seen under an inclination of $\sim 60^{\circ}$, extending out to $100 \mathrm{AU}$ and exhibiting a mass accretion rate of $9 \times 10^{-6} M_{\odot} \mathrm{yr}^{-1}$. Our study suggests that the spectro-interferometric capabilities of the latest generation of long-baseline interferometric instruments are particularly well suited to reveal the contributions from active accretion processes taking place close to the star. 


\section{References}

Akeson, R. L., Ciardi, D. R., van Belle, G. T., Creech-Eakman, M. J. \& Lada, E. A. 2000, ApJ 543,313

Dullemond, C. P., Dominik, C. \& Natta, A. 2001, ApJ 560, 957

Eisner, J. A., Lane, B. F., Hillenbrand, L. A., Akeson, R. L. \& Sargent, A. I. 2004, ApJ, 613, 1049

Hernández, J., Calvet, N., Briceño, C., Hartmann, L., \& Berlind, P. 2004, AJ 127, 1682

Hillenbrand, L. A., Strom, S. E., Vrba, F. J. \& Keene, J. 1992, ApJ 397, 613

Kraus, S., Preibisch, Th., \& Ohnaka, K. 2007, ApJ, submitted

Lynden-Bell, D. \& Pringle, J. E. 1974, MNRAS, 168, 603

Leinert, C., van Boekel, R., Waters, L. B. F. M., et al., 2004 A $\& A, 423,537$

Men'shchikov, A. B., \& Henning, T. 1997, A\& A, 318, 879

Millan-Gabet, R., Schloerb, F. P., \& Traub, W. A. 2001, ApJ 546, 358

Millan-Gabet, R., Malbet, F., Akeson, R., Leinert, C., Monnier, J., \& Waters, R. 2007, Protostars and Planets V, eds. B. Reipurth, D. Jewitt, K. Keil, University of Arizona Press, p. 539

Monnier, J. D. \& Millan-Gabet, R. 2002, ApJ 579, 694

Monnier, J. D., Millan-Gabet, R., Billmeier, R., et al., 2005, ApJ 624, 832

Muzerolle, J., D'Alessio, P., Calvet, N. \& Hartmann, L. 2004, ApJ 617, 406

Natta, A., Prusti, T., Neri, R., Wooden, D., Grinin, V. P., \& Mannings, V. 2001, A\& A, 371, 186

Ohnaka, K., Driebe, T., Hofmann, K.-H., Leinert, C., Morel, S., Paresce, F., Preibisch, T., Richichi, A., Schertl, D., Schöller, M., Waters, L. B. F. M., Weigelt, G., Wittkowski, M. 2006, A\& $\&$ A 445, 1015

Preibisch, Th., Kraus, S., Driebe, T., van Boekel, R., \& Weigelt, G. 2006, A\&\&A, 458, 235

Pringle, J. E. 1981, ARA $\& A$ A, 19, 137

Polomski, E. F., Telesco, C. M., Piña, R., \& Schulz, B. 2002, AJ, 464, 1

\section{Discussion}

ARDILA: Do you include in your modeling absorption by gas located inside the dust sublimation radius?

KRAUS: We agree that full radiative transfer modeling, including gas absorption, would provide a more complete picture. However, including dust and gas opacities in one model would dramatically increase the complexity of the radiative transfer modeling, which was out of the scope of our investigation. Based on the results of the theoretical work by Muzerolle, D'Alessio, Calvet, et al. (2004), which predict that the gaseous disk is optically thick and geometrically thin, we expect gas absorption also to be a secondary effect.

CABRIT \& NATtA: What are the reasons to include an extended envelope? Would it be possible to fit the data with a pure disk geometry?

KRAUS: We required an extended envelope to successfully reproduce the pronounced shape of the MWC 147 mid- to far-infrared SED and in particular the sharp drop at $15 \mu \mathrm{m}$ in the Spitzer/IRS spectrum. We were not able to reproduce the SED equally well assuming only a disk component. The presence of an extended envelope is also supported by the mid-infrared imaging presented by Polomski, Telesco, Piña, et al. (2002), revealing an elongated diffuse emission component, extending $\sim 6$ arcseconds around MWC 147 . 